\title{
Remote Access of Computer Controlled Experiments
}

\author{
doi:10.3991/ijoe.v4i4.704 \\ K. Nilsson, J. Zackrisson and M.I. Pettersson \\ Blekinge Institute of Technology/ School of Engineering, Ronneby, Sweden
}

\begin{abstract}
-in this paper, we present a way for students to access and operate laboratory equipment, controlled by a laboratory computer via a remote access program. In this way, the solution is not dependent on the specific laboratory equipment, as long as the equipment can be remotely controlled. The system can easily be altered to be used in another laboratory setup. Students are able to make reservations of experiment sessions through a web interface, which is administrated by the system administrator. The solution proposed in this paper is one way to speed up the development of remote accessible laboratories. Most of the proposed solution is based on open source software and the hardware is built on ordinary consumer parts, which makes the proposed remote laboratory architecture cost effective.
\end{abstract}

Index Terms-Remote handling, Student experiments, Training, e-Learning

\section{INTRODUCTION}

The purpose of this work at Blekinge Institute of Technology (BTH) is to provide an effective and efficient environment for experiments. This paper is focusing on antenna equipment and how to remotely control this hardware. The design allows students to perform experiments around the clock, every day. The strength of the system is the access to expensive equipment without the need of a supervisor, continuously monitoring the experiment. This helps meet the demands of more students without increase in staff and funding resources. According to [1] the number of students is increasing compared to the funding dedicated for hands-on experiments. In engineering education, a key-activity to improve the learning process is hands-on experience, gained from laboratory experiments [2].

Students want extended accessibility and free access to learning resources, this demands more flexible laboratories that not only are physical accessibility around the clock. They must also be accessible without the geographical limitation. Today there are many academic institutions offering web-based experiments, so called remote laboratories, which are supporting remote access of physical hardware $[3,4]$. The architecture presented in this paper is describing a general solution for how a computer-controlled experiment can be made remote accessible, without the need for special hardware or customized software. The presented architecture is built on simple and robust hardware running standard software.

\section{General Structure}

The laboratory system is divided in three major parts, Figure 1. The first part is the Web server. The second part is the controller. The last part is the computer the students will remote control to perform experiments. Through the laboratory's web interface, students can allocate resources and mange their experiments. To get access to the laboratory students must first authenticate themselves. This is done through a login webpage where the student enters his/her email address and password. The first time a student want to use the system, the account has to be activated. This activation is generating a password and which is sent to the users email address. When a student is logged-in, he/she can make a reservation. The student can choose which system installation to use. A system installation is a snapshot of hard drive content, also called clone, and consists of an Operating system and all the tools needed for the experiment. The controller is handling and distributing these clones of different operating systems and software. A Power Distribution Unit (PDU) is handling the power distribution to all of the computers. For the cloning system to be able to install a new operating system the remote controlled computer (RCC) have to be rebooted. A PDU that switch the power off and on are the most reliable solution of ensuring that the computer is rebooted.

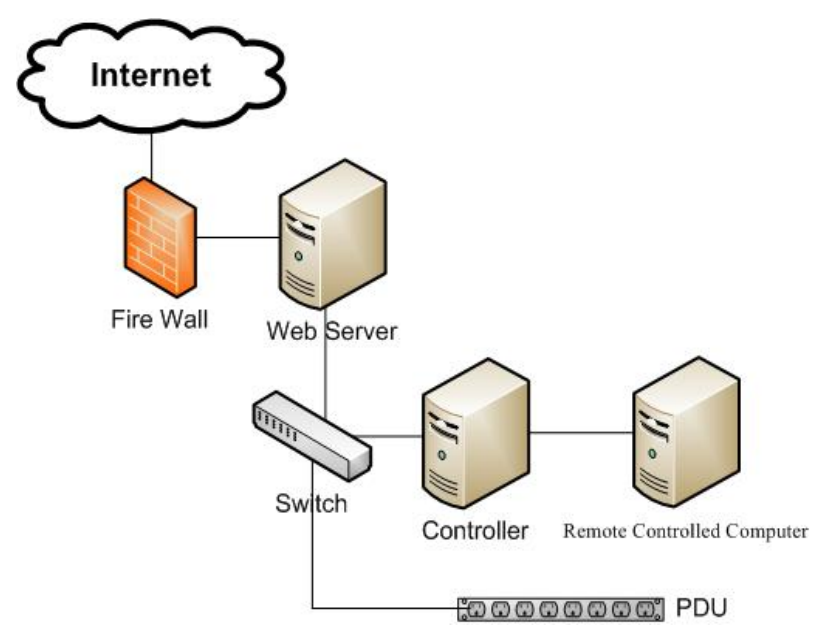

Figure 1. General view of the core parts in the system.

Control mechanisms make sure that it is safe to give full access of the remote controlled machine to the student. That means that they do not require supervision during their experiment. Users are given full access to the laboratory computer and have the freedom to alter the entire operating system. This is made possible due to the cloning system. When a laboratory session is over, the cloning system will wipe the RCC's hard disk content and install a new clone for the next experiment. 
To prevent abuse of the system the only accepted data communication is from the student that booked the laboratory session this security measure is preventing the system from being used for adverse activities.

Both the web server and the controller are running on FreeBSD [5], an open source operating system.

\section{A. Web Server}

The web server is handling the web interface, where users can make experiment reservations. In the antenna laboratory this computer are also acting as a firewall and a DNS server.

The Web Server is providing the following services:

- Firewall (PF)

- DNS

- $\mathrm{DHCP}$

- Web server (Apache)

○ Web application

Remote access is vital to the laboratory. At the same time, security measures must be taken to guarantee that the system is not used in adverse ways. A way to provide remote access is to let a firewall give certain IP-addresses access to the laboratory. When a student chooses to begin a laboratory session in the web interface, the address is saved. The IP-address is then used to generate firewalls rules that only allowing data traffic between the user's computer and the RCC in the laboratory. After the laboratory session is over, access to the laboratory is disabled. This solution requires that the student have the same IP-address for the whole laboratory session. Depending on the demands of the experiments, a system administrator can make exceptions and e.g. grant access from the RCC to different web sites.

\section{B. Controller}

The system used for creating clones is the EXP experiment system [6]. It is provided by EXP innovation and is used under a restricted BTH license. The experiment system EXP is handling all of the clones that can be installed on the RCC. Clone images are raw copies of the content off a hard drive. All of the hardware is built on standard PC parts with this specially built EXP software, which runs on the controller. The controller is acting as a server to all of the RCCs in the network.

The cloning system is providing the following functions:

- Booting a system on the RCC

- Creating of system clones

- Installing of system clones

- Cleaning hard disks

The system administrator creates clones by installing all of the necessary software on the RCC and by using the cloning software to make a complete clone image of its hard drive. The cloning process is providing the necessary tools for experiments without the need of further installations during the laboratory sessions. Because most experiments don't need to use the whole hard drive a Microsoft Windows XP operating system image can be installed on a 10GB partition and the clone tool can be set to only clone this $10 \mathrm{~GB}$ area. To compress the size of the clone even more the run length encoding (RLE) compression can be used.

All of the software, except the cloning system, is released as open source software and can downloaded from http://svn.openlabs.bth.se/trac/openlabsweb

\section{Remote Controlled Computer}

The RCC are a built on hardware that are suited for the type of operating system that are demanded by the experimental hardware. In this case are the RCC built on ordinary PC parts. This computer is the only part of the system that is accessed by the student; the controller is transparent for the end user and the web server are only access by the web interface.

\section{Power Distribution Unit}

The PDU are distributing power to the computers in the laboratory. The PDU can be controlled via SNMP. When a RCC is cleaned, cloned or reinstalled with a new clone, a reboot of the computer is needed. There are different software solutions for rebooting the computer, but only ways to make sure that the computer is rebooted is to turn the power off and on again. This approach demands that the hardware is supporting power up after power failure. The benefits of a PDU compared to other solutions are that it is independent of hardware manufacturers and are not demanding operating system integration. Unfortunately, it put some extra strain on the RCCs' hardware as the power is cut without notification.

Older hard drives had problems with start-stop (CSS) cycles, which happens when there is a power failure or in our case cut the power to the hard drive without notification. Modern hard drives have different ways to handle power failure. One solution is CSS landing zones, which are specially prepared regions on the platter to where the read head automatically returns in case of a power failure. One other solution is moving the read head away from the platter [11] in case of a power failure. Most of the new hard drives on the market are guaranteeing a start-stop cycle count of $50000[12,13]$ or nearly 3 years (1000 days) of operating at 50 cycles per day.

\section{SYSTEM MANAGEMENT}

The solutions presented so far focuses on the general structure of a remote laboratory that can be used with various types of hardware and or software. One of the laboratories adapting this general structure is the antenna laboratory [9]. The antenna laboratory is managed from a web application running in a web browser, where the user can make experiment reservations, deciding when, and what type of experimental environment to use. The administrator can create course occasions for different courses, and the system resources can be distributed among these occasions and shared among different courses. Course occasions are limited in time and the number of users it can handle. This is an estimation of how many reservations that can be expected. For an occasion there can be several teachers assigned and it is up to them to do further management of the occasion. To give access to the system an administrator or teacher has to register the e-mail address for the users. The first time a user wants to access the system, the account has to be activated. This makes the system send an automatically generated password to the users e-mail address. There are 
three different levels of access to the system, administrator, teacher, and student. Depending on the role, they will be presented with different options.

\section{A. Administrator}

The administrator is responsible for the general management of the system; he/she is creating courses and deciding the limitations of recourses for this course. It is the administrator that is creating the different clones that later on can be used in the courses. The system are built in a hierarchy, the administrator are overruling both the teacher and the students settings.

\section{B. Teacher}

The top part of the teacher interface in Figure 2 is showing information about the course occasion. Teachers can make pre-reservation, e.g. for teacher-lead experiments. This type of reservation is given priority over ordinary reservations and do not have the time restrictions ordinary reservations have. The bottom part of the teachers interface Figure 2 is for managing users in the courses occasion. All of the users registered to this occasion are presented with their e-mail address and user type. In the bottommost area e-mail address of new users can be added, this field is accepting multiple inputs as long that there is a new line between every e-mail address.

The teacher can perform the following actions:

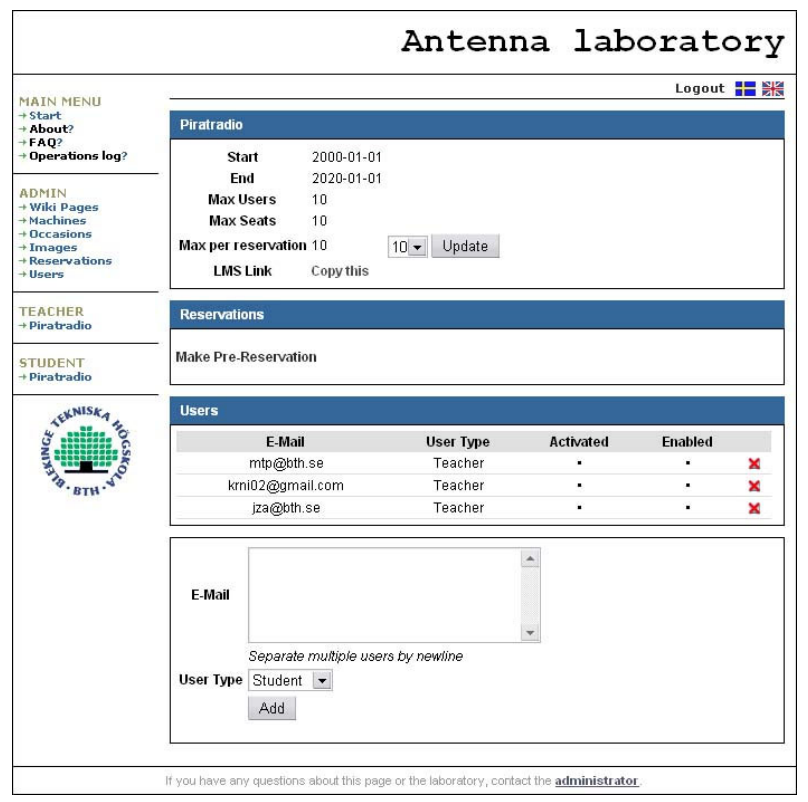

Figure 2. Teacher interface.

- Define the number of computers a student can reserve.

- Make a pre-reservation.

- Add and remove users from the course.

\section{Student}

Students are able to make reservations of laboratory session. It is up to the administrator to decide the maximum duration of one laboratory session and decide which clones that are allowed for the session. Figure 3 shows an overview of the reservation system. The information given is the number of RCC that are available every hour. In the antenna laboratory, there is only one computer and therefore the maximum numbers of accessible RCCs shown in Figure 3 is one.

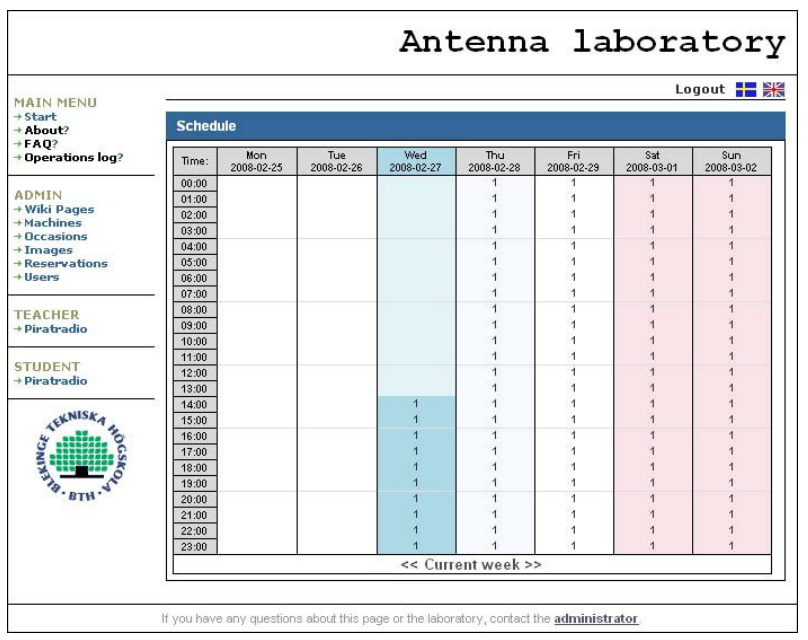

Figure 3. Overview of the reservation page.

The reservations are made simply by clicking on an available RCC. After a reservation is made, the cloning system needs time to transfer the clone from the controller to the RCC. Depending on size of the clone, variations in cloning time is hard to approximate and therefore a onehour preparation period is added to the reservation system to guarantee that the system have finished. Five minutes before the experiment begins, an e-mail is sent to the student an as reminder. This massage contains all the information needed for the experiment, such as user name, password, and IP-address.

\section{ANTENNA LABORATORY}

In the antenna laboratory, the main part of the software used in web server and controller was developed for the security laboratory at BTH [11]. The security laboratory is focusing on security and the possibility to experiment with dangerous software in a controlled environment. In the antenna laboratory, the focus is to give students remote access to expensive equipment. Figure 4 is illustrating the structure of the antenna laboratory. The antenna laboratory is expanding the general configuration, shown in Figure 1, with one web camera, one camera accessed over the USB and the antenna equipment.

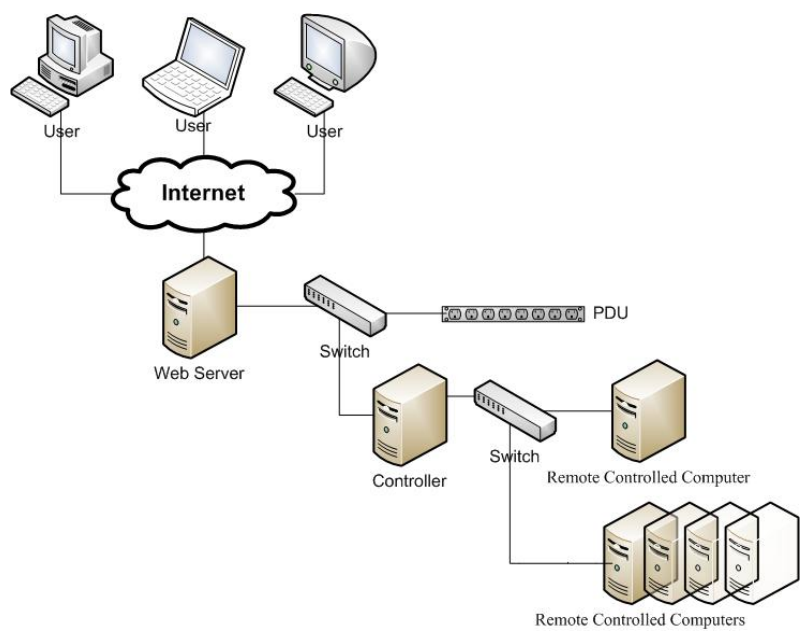

Figure 4. Expanded system with more homogenous RCC. 


\section{A. Antenna Equipment}

The equipment used in the laboratory is provided by Lucas-Nuelle Lehr- und Messgeraete GmbH [7]. The company has a wide range of products for antenna laboratories. The one used in this work is the "TAT Antenna Techniques" [8]. This equipment makes it possibly to do experiments in antenna technology fundamentals and is recommended for basic courses in antenna theory. The laboratory equipment is communicating by USB. Without the protocol specification for the USB controlled device, it is almost impossible for third party software to access the hardware. This means we have to depend on the provided software, and that is the reason behind this remote control solution.

\section{B. Cameras}

In the antenna laboratory, there are two cameras. One is used for monitoring and to give visitors of the laboratory home page an overview. This camera is continuously taking pictures of the laboratory equipment and they are shown at the home page [9]. The second camera is used during the experiments in the laboratory. This camera is covering two important areas; one is to provide a feeling of working with real equipment and the other are to give necessary information for the laboratory assignment. The camera that is used by the student is remotely adjustable in vertical and the horizontal level. Depending on the bandwidth of the students Internet connection, the image quality can be adjusted, to allow everyone the same opportunity to perform experiments.

\section{Preforming a Experiment}

To remote control the antenna experiment, Microsoft Remote Desktop is used, which is part of the Windows operating system. When the student confirms that he/she is ready to start the experiment session, the firewall settings are updated automatically to allow connections to the RCC. The firewall will then be accepting incoming and outgoing traffic to and from the users IP-address. The student is then allowed to connect to the remote controlled computer. Figure 5 shows the system running a laboratory session.

During the session, students can perform all of the tasks that an ordinary laboratory can offer. In the Figure 5 to the right is the software program for the TAT Antenna Techniques [8] and to the left is the remote controlled camera.

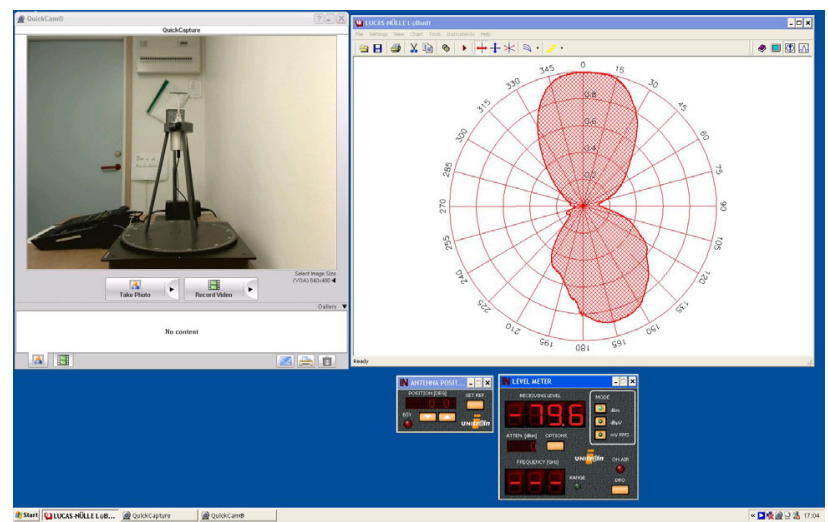

Figure 5. Running laboratory session, to the right is the software program for the tat antenna techniques and to the left is the remote controlled camera.

\section{AdAPting the System to NeW ApPlications}

The system can be adapted to handle different types of laboratory software or hardware, as long as it is controlled by a computer that allows remote control. The requirements (of the laboratory equipment) are that a computer controls it and that the operating system is supporting remote access.

The system can for example be used for remote accessing:

- Expensive or fragile hardware

- Sensitive or expensive software

- Sensitive or dangerous hardware

One of the advantages of the system is the freedom for the user; one user can control all of the hardware and software resources without worry about the next user. Regardless of what type of changes that has been made in the software, the system always restores the software between every laboratory sessions. The flexibility allows the system to be adapted in various ways.

\section{A. Scaling}

Depending on the needs and demands of the system, more RCC with homogenous hardware can be added. One important thing to consider when expanding the laboratory with more RCC is the cloning time, depending on the LAN configuration and the number of simultaneous cloned computers the cloning time can increase. The system used today is supporting feedback from the RCC when a successful clone is ready but the software handling this feedback is still under construction. That means, a fixed preparation and cleaning time must be used, which have to be estimated by hand. Figure 6 is illustrating a scaled version of the antenna laboratory.

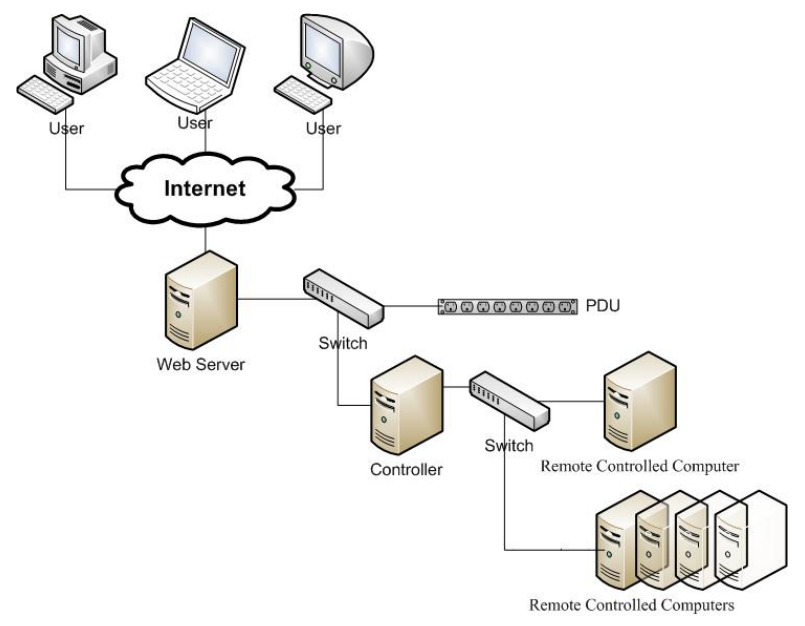

Figure 6. Expanded system with more homogenous RCC.

\section{B. Adapting to a Specific Problem}

On BTH the EXP system are been used to supply on campus laboratories with on demand reinstallation of the entire operating system. Different laboratory sessions demand different software and sometimes even different operating systems. Instead of having multiple operating systems installed on the local computer, the teacher can prepare a clone in advance. This clone is then installed on the local computers. By using this solution the teacher only needs to maintain one copy of the software and 
students don't have to worry about system configuration, all of the computers are having the same configuration when the laboratory session are starting.

\section{CONCLUSION AND FURTHER WORK}

In this paper, a general computer based structure is presented that can be applied to various computer based experiments. The proposed solution has the potential to be adapted to a wide range of laboratory experiments The laboratory could also be used to give students access to expensive and licensed software (if the end user license agreement allows it), without need to install it on their home computers. The solution of the antenna laboratory is focusing on the access of the computer through for example remote desktop rather than focusing on the interface controlling the hardware. This approach makes detailed knowledge of the communication between hardware and the computer unnecessary. The solution is a fast and simple way of constructing a remote accessed laboratory. The goal is to offer access to experimental equipment around the clock and the experience of a genuine laboratory. Adapting this architecture will help the universities to increase remote experiment possibilities, without increasing running cost per student.

BTH has developed software for online laboratory that uses the same equipment as a traditional laboratory. In the antenna laboratory the difference between a traditional laboratory and the online laboratory are the ability to change antennas in the laboratory equipment, this limitation are going to be further investigated.

\section{REFERENCES}

[1] Magin, D, and Kanapathipillai, S, "Engineering Students'Understanding of the Role of Experimentation", European Journal of Engineering Education, Vol. 25, no. 4, 2000, pp. 351-358 (doi:10.1080/03043790050200395)

[2] Ursulet, $\mathrm{S}$ and Gillet,D, "Introducing Flexibility in Traditional Engineering Education by Providing Dedicated On-line
Experimentation and Tutoring resources", the International Conference on Engineering Education, Manchester, UK, August $18-21,2002$

[3] Nedic, Z and Machotka, J, "Remote Laboratory Net-Lab for Effective Teaching of 1st Year Engineering Students", Proceedings of the REV 2007 Conference, Porto, Portugal. June 25-27, 2007

[4] Scapolla, A, Bagnasco, A, Ponta, D, and Parodi, G, "A Modular and Extensible Remote Electronic Laboratory", International Journal of Online Engineering, Vol. 1, No. 1, 2005

[5] http://www.freebsd.org/

[6] Mellstrand, P, "Informed System Protection", Dissertation, 2007, pp. $163-178$

[7] http://www.lucas-nuelle.de

[8] http://www.lucas-nuelle.de/299/Products/Training_Systems/Infor mation_and_Communication_Technology/tan.htm

[9] http://antenna.openlabs.bth.se/

[10] Zackrisson, J and Svahnberg,C," OpenLabs Security Laboratory The Online Security Experiment Platform" International Journal of Online Engineering, Vol. 4, 2008

[11] Patricia Kim and Mike Suk, "Ramp Load/Unload Technology in Hard Disk Drives" whitepaper, 2007.

[12] Maxtor Corporation, "Maxtor DiamondMax 10" specification, 2006.

[13] Hitachi Global Storage Technologies, "Hitachi Deskstar 7K1000" specification, 2007.

\section{AUTHORS}

K. Nilsson is with Blekinge Institute of Technology, Ronneby, Sweden (e-mail: kristian.nilsson@bth.se).

J. Zackrisson is with Blekinge Institute of Technology, Ronneby, Sweden (e-mail: johan.zackrisson@ bth.se).

M.I. Pettersson is with Blekinge Institute of Technology, Ronneby, Sweden (e-mail: mats.pettersson@ bth.se).

The VISIR project is supported by VINNOVA (Swedish Governmental Agency for Innovation Systems). Manuscript received 24 October 2008. Published as submitted by the authors. 